\title{
Use of atmospheric modelling for the territorial planning of technological structures
}

\author{
G. Genon, E. Brizio \& M. Poggio \\ Turin Polytechnic, Italy
}

\begin{abstract}
The introduction and distribution of transport activities, energy infrastructures and technological plants throughout the territory are operations that must be conducted taking into consideration some constraints or objectives, and that of respecting environmental quality and compatibility seems to be increasingly more important, in particular with reference to the atmospheric transport of gaseous emissions. On the basis of defined emission scenarios, corresponding to hypothesised technological conditions, it is necessary to evaluate the loads of the emitted pollutants and then to evaluate the modifications of the air quality on the basis of atmospheric transport mechanisms. In order to obtain such results, it is necessary to use the correct instruments to first of all evaluate the value of the emissions and then to describe the atmospheric transport in a reliable way and to establish the damage in terms of externalities or effects on human health. In order to define correct territorial planning instruments, different scenarios derived from different location or technological choices must be compared. Three examples are given to illustrate such an approach:

- optimal collocation of an incinerator for municipal solid waste, taking into consideration both emission and impact aspects and the benefit that can be derived from the elimination of the environmental load due to conventional thermal and electric systems that are substituted by new systems;

- evaluation of either concentrated or distributed cogeneration systems, in consideration of the different potentialities, technological configurations and spatial distributions of the different possibilities;

- verification of the effect of interventions to limit urban vehicle traffic or of a different road distribution on the quality of the air.

Some results that were obtained for the three aforementioned cases for the Piemonte Region are presented here, but the main purpose of this article is to show the necessity of using this type of approach as an aid to territorial choices.

Keywords: atmospheric pollution, territorial planning, models, externalities, energy.
\end{abstract}




\section{Introduction}

The introduction of large technological structures or flows of vehicle traffic into the texture of metropolitan areas is an operation that is increasingly more subject to resistance and preoccupation by the local populations, in particular concerning the foreseen atmospheric pollution and the changes to the quality of the air. Even though the role of technology is surely important in setting up ever more efficient impact control systems, these instruments should however be accompanied by a careful evaluation of the environmental incidence; each single intervention should therefore be evaluated taking the environmental constraints into account and adopting methodologies that allow estimations to be made of the effective environmental result that the coupling of technological solutions and the receptive capacity of the surrounding environment involves. The decision makers, at various territorial levels, must be put in a position where they can recognise what the results of different technological scenarios or different localisation options might be and then to be able to use such information as part of the set of considerations that make up the substratum of territorial planning.

Sufficiently developed and precise forecasting calculation instruments nowadays exist that can be used as an aid in such evaluations and it is on the capacity of using such instruments that this paper is concentrated, while presenting some detailed results relative to particular situations and trying to draw more general considerations of a methodological type from these results.

\section{Definition of emission factors}

The starting point for any estimation of territorial impact is the knowledge of the actual atmospheric type pollutant flow that is emitted from a determined technological structure; in the absence of such quantitative information it is in fact not possible to carry out a concrete estimation of the changes in the quality of the air and consequently of the possible risks or damage. It is usually difficult to have direct evidence of emissive flows that one can deal with, either because we face forecasting evaluations, and the technological object whose emissions one intends to measure is not yet available, or because one intends studying a phenomenon that involves numerous sources arranged throughout the territory, whose analytical quantification is extremely difficult to put into practice.

In these situations it is necessary to make use of forecasting methods based on emission factors, that is to say, on numerical indications that are able to correlate the quantity of emitted pollutant to a specific magnitude that characterises the dimension of the emitting source; as such a variable is known or fixed in the particular application, an at least approximate knowledge of the emissive flow can be derived from the use of the emission factor.

In order to clarify this concept, mention can be made to some examples of such factors: the quantity of carbon dioxide emitted per unit of energy generated by a thermoelectric plant, the flux of thin powders dispersed in the air per volumetric unit of heated houses, the quantity of solvent dispersed in the air due to painting operations from a unitary quantity of raw material. 
The thus schematised emission factors are functions of various magnitudes, that is:

- the raw materials that are used

- the technological process that is considered

- the operative conditions from the process and plant engineering point of view

- the pollutant removal systems

and they can undergo large variations due to the specific situations; in some cases they can be estimated through hypothesis of mass and heat balance, while in other cases, a definition can be made in a statistical manner starting from experimental type indications. The topic of the estimation and verification of emission factors, given the importance of this knowledge and interpretation instrument able to evaluate and forecast the impacting significance of determined technologies, has been dealt with for some time now by the most important environmental agencies at a worldwide level (U.S. E.P.A., E.E.A, European IPPC Bureau, national environmental agencies) and reviews and inventories are periodically published on this matter. On the basis of such data, it is therefore possible to consider that the estimation of the generation of pollutants, which, from a determined situation of the scenario of the sources is destined to derive, can be considered sufficiently reliable.

\section{Modelling of the atmospheric modelling and externalities}

The next step in the described study consists of the quantitative definition of the correlation between emitted pollutant flows and changes in the quality of the air, and more precisely, the increase in the concentrations of the pollutants that can be found in determined and significant points and in each moment in the area surrounding the emitting source.

From the point of view of the phenomenological modelling of the thus induced correlation, it should be recalled that different factors can be influent, that is:

- the modality of the emission

- the meteorology of the receiving environment

- the surrounding orographic situation

- material exchange and transformation phenomena in the atmosphere

- the receiving characteristics of the ground.

A great deal of literature exists on describing the physical, chemical and physic-chemical phenomena that subtend such factors and on how to use this knowledge in the preparation of mathematical models to use as calculation instruments that must be calibrated to the specific situations; models of different complexity and facility of use are available, of different specificity for particular environmental situations or for different types of pollutants with different environmental destinations, for different temporal or spatial horizons on which the forecasting knowledge of the levels of concentrations, starting from the flows, must be practised. It is not the intention of this work to make a summary collection of this very rich literature, but rather to point out that it is possible to find models that couple a good rigorousness of methodology and significance of 
representation with practical applicability, that above all involve acceptable calculation times and specific site knowledge, especially from the meteorological point of view; their use will subsequently be demonstrated.

The scenario of the calculated air concentrations is in itself a useful result, making it possible to compare the impact with standards of predetermined environmental quality, in order to allow defined uses of a resource (in this particular case that of the atmosphere); but it seems to be very important, in order to estimate the real acceptability or negativity of a determined polluting situation, to correlate the levels of environmental concentrations with the frequency of hygiene-sanitary damage to humans and possibly to translate such damage into monetary terms.

A significant amount of international experience also exists concerning this aspect, in particular with the purpose of defining two aspects:

1) the identification of the number of cases of various pathologies connected to defined levels of quality of the air and statistical interpretation of the results;

2) the econometric evaluation of the costs for the public operator of a single pathology, from the point of view of the costs due to lost activity or due to effective necessity of medical treatment.

It is not intended to report here the large amount of literature on these two aspects in detail, but to underline that this is a consolidated methodology based on statistical results obtained from different sources, though with the inevitable defects of numerical discrepancy; the application of the method will be the subject of some examples reported in the following chapter.

\section{Comparison of scenarios}

In the practical application of territorial planning events, the problem often arises of the comparison of scenarios, by this we mean the different technological solutions, different hypotheses of location, different hypotheses of production or supplies of energy flows (electricity and/or heat). While the definition of polluting flows corresponding to different design hypotheses is basically an already acquired knowledge patrimony, how these source factors can be compared still has to be studied in depth to offer the decision makers a correct and practically usable basis of environmental comparison. The adoption of the previously schematised approach, made up of the use of emission factors, from the reconstruction of the scenario of quality corresponding to a determined situation, from the aggregated evaluation of the risk or damage corresponding to a determined environmental situation, seems to be the correct way of arriving at a quantisation of the environmental effect of a determined hypothesis.

A procedure of this type should obviously come after a verification of the minimum plant capacity requirements of reducing the emissions that each evaluated system should possess, as this seems to be a minimal, irremissible requirement that should however guarantee the adoption of the best technology for the considered systems. However, after this, as there exist comparison hypotheses, dealing more with environmental compatibility, it is on the evaluation of this compatibility that the described approach is used. 
The considered operation is surely complex as far as acquiring process, environmental and territorial information, the necessity of calibrating predictive models on dispersion or atmospheric destiny, and the complexity of the calculation especially in complex territorial structures or with a multiplicity of sources is concerned; anyway these obstacles must be overcome, in view of the result that can be obtained. The procedure, in light of the aforementioned aspects of complexity, must basically be directed to evaluate cases with large environmental loads, where the best reduction technology does not seem enough and it is therefore necessary to assess the compatibility on the territory.

\section{Examples of application of the methodology}

\section{Example 1: localization of a MSW incinerator}

The city of Turin planned to construct a MSW incineration plant, with a potentiality of about $1200 \mathrm{t} / \mathrm{d}$; once the size and the technology were decided, the flows of the pollutants into the atmosphere and the thermal and electric power that could be used outside the plant were calculated. As far as the pollutants are concerned, it is of fundamental importance to understand the effect on the quality of the territory, while for the thermal power, it is very important the local energy network and the possibilities of transferring heat to local district heating existing systems; the consequent substitution of conventional sources and the elimination of the corresponding loads represent an environmental benefit.

Three possible sites of the metropolitan territory were considered and the topographic situation, the local meteorology, the possibility of energy transfer, the conventional substituted sources and the consequent benefits in terms of environmental load elimination were evaluated for each of these sites.

The fallout maps of the main pollutants (dust, nitrogen oxides, micropollutants) were drawn up for the three locations using a Gaussian type forecasting model (the AERMOD model by U.S. EPA [1]) and the concentrations were evaluated through a comparison with environmental standards (an example of a map is shown in Figure 1, taken from [2]); once the acceptability was showed for each of the sites, the possibility of setting up an energy network, thus contributing to the metropolitan energy production, supplying the district heating network and substituting individual domestic heating plants was then examined; the latter two aspects involve the elimination of environmental loads that at present exist.

After evaluating the present and future environmental situation and translating the environmental loads into externalities, through consolidated correlations for a metropolitan territory (the ExternE methodology, [3]), it was possible to establish either an increase or decrease of the externalities for each site and this verification made it possible to establish a scale of priorities, from this point of view, of the three territorial situations that were considered.

Table 1 (taken from [4]) illustrates the results, in terms of externalities, of such a comparison. 
204 The Sustainable City IV: Urban Regeneration and Sustainability

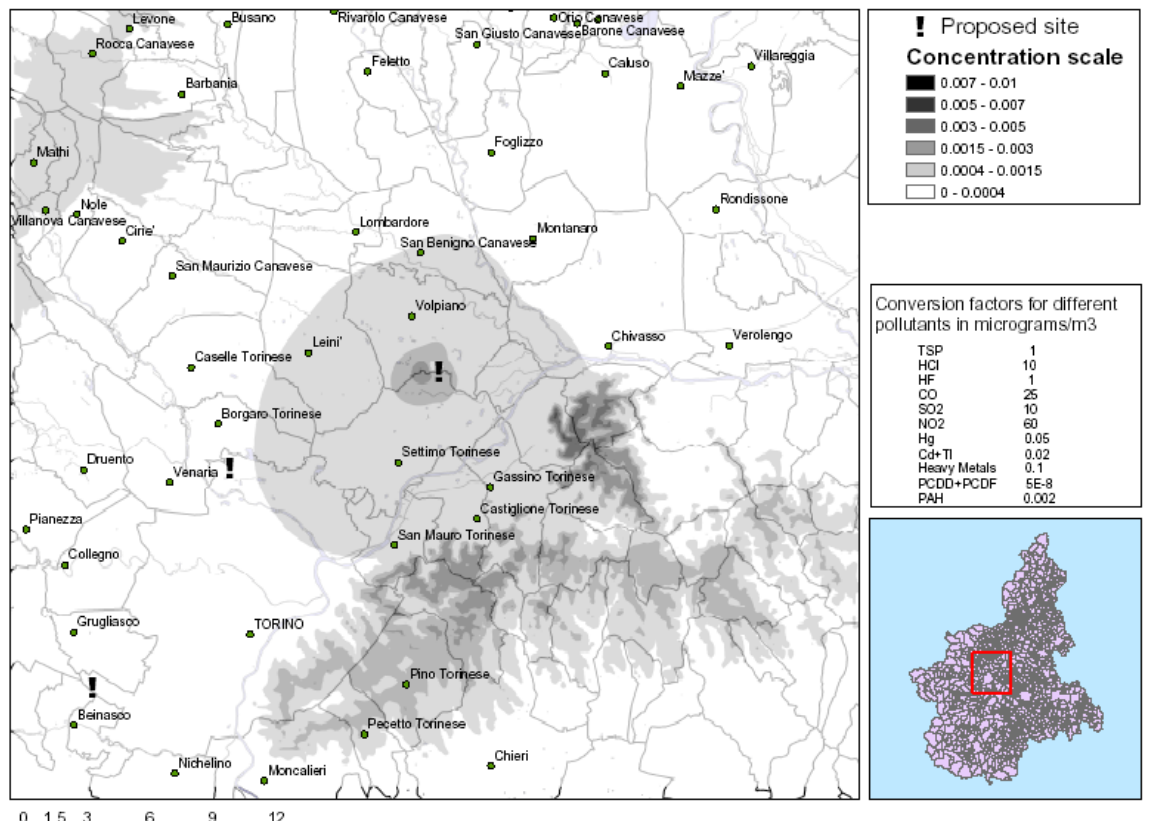

Figure 1: $\quad$ Isoconcentration map for the incinerator placed in Volpiano.

Table 1: $\quad$ Environmental balance for the three localizations.

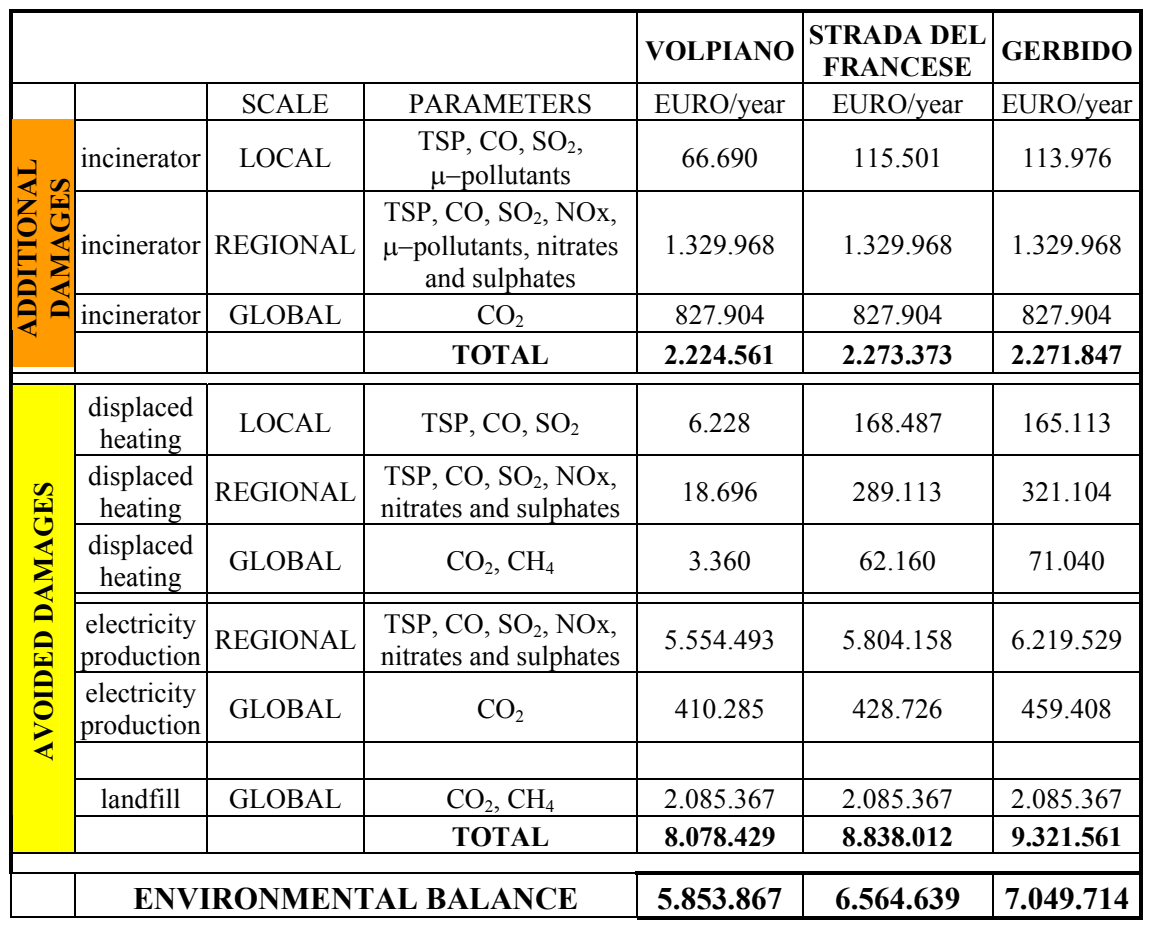


Example 2: evaluation of a cogeneration hypothesis

A proposal was made, in a town of about 30.000 inhabitants, in Piedmont, to construct a cogeneration type electric production plant capable of producing electric energy to use in the energy network and, at the same time, to produce heat that could be used in a district heating system for an urban area; on the basis of data concerning potentially connectable thermal utilisation and of the configuration of the proposed cogeneration system (about $30 \mathrm{MW}$ of thermal necessity, and cogenerated electric energy for the network), the proposal of installing a plant made up of engines (10MW) and boilers (40 MW) was examined. It seemed important to evaluate the compatibility of such an installation and the significance in terms of environmental effects [5].

The loads of the main pollutants introduced by the new system and those that would be substituted are shown in Figure 2. While the result for the carbon dioxide can immediately be used, as the effect of this pollutant is not local, and as it is significant to directly compare the emitted flows to estimate an environmental balance, for the other pollutants (nitrogen oxide, sulphur oxide, dust) as in the previous case, it was necessary to evaluate the fallout maps, taking into consideration the newly introduced loads and those eliminated in order to verify where to find benefits or deteriorations on the territory and of what entity. Figure 3 shows an example of a map concerning the differences in concentrations between the present situation, before the intervention, and situations that can be hypothesises for after the intervention.

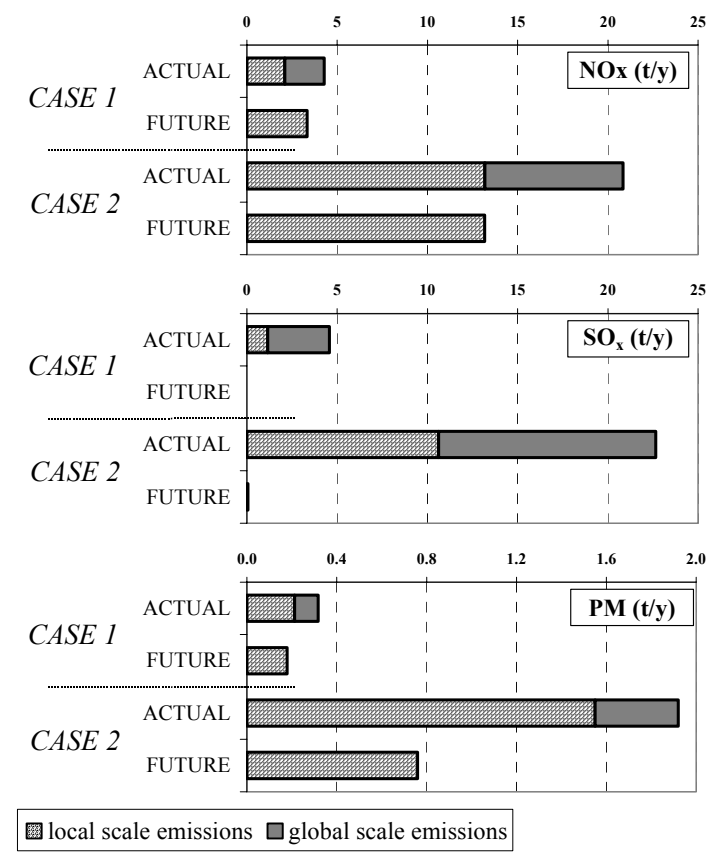

Figure 2: $\quad$ Environmental loads before and after the intervention. 


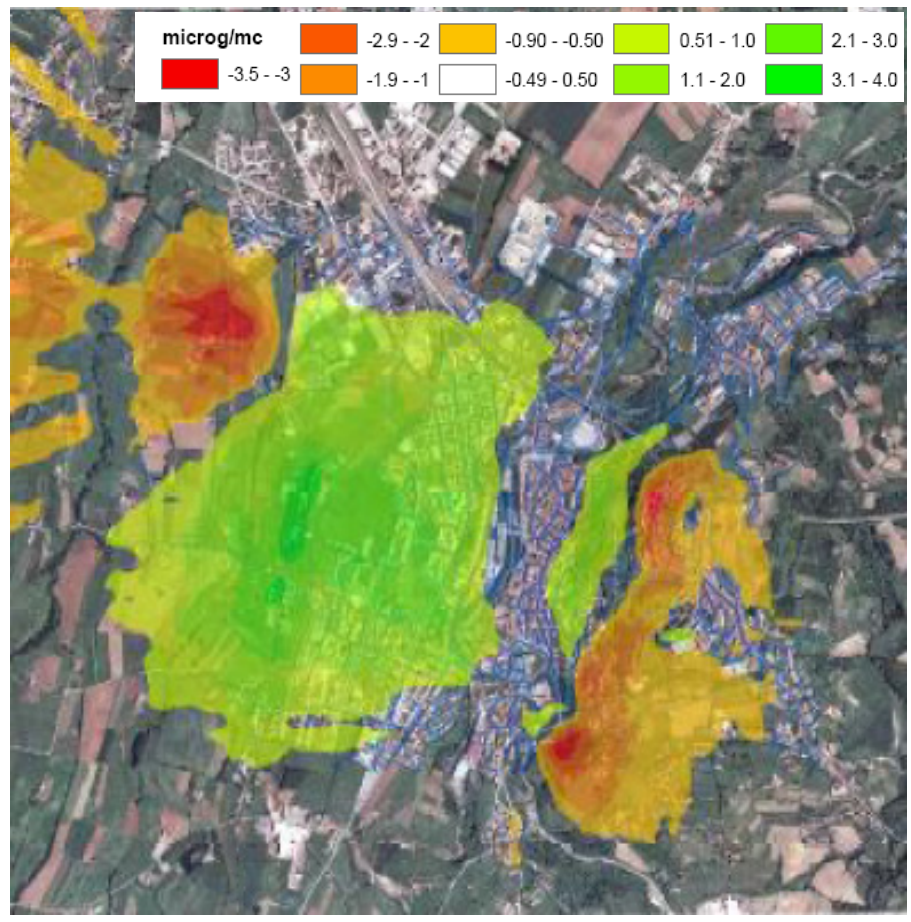

Figure 3: Difference of concentrations between after and before the intervention.

The two types of comparison previously described (the first global and the second local) made it possible, in the specific case, to show the sure general benefits of the initiative, but with a certain local impact in the zone immediately surrounding the plant. The verification of acceptability of such a degrade on the particular site, and the weight that should be comparatively given to such a burden with respects to the general benefits is an aspect of political choice of territorial planning; the data that was supplied are certainly a necessary aid for this operation.

\section{Example 3: effect of the limiting of urban traffic}

The city of Cuneo (about 60.000 inhabitants) is evaluating the effect of limiting traffic in some central zones on the air quality in order to establish the opportunity of defining new limitations.

So as to carry out this kind of evaluation, described into details in [6], it was first of all necessary to set up a modelling instrument that was able to correlate traffic flows (and their emissions that could be evaluated on the basis of known specific factors of emission of the vehicular fleet) with the quality of the air; such an instrument needs to take into consideration particular situations concerning the geometrical structure of the town, the wind field and the 
turbulence at a ground level. A model that had already been used in other urban areas [7] was verified and calibrated according to the specific territorial requirements (an example of such a calibration is given in Figure 4).

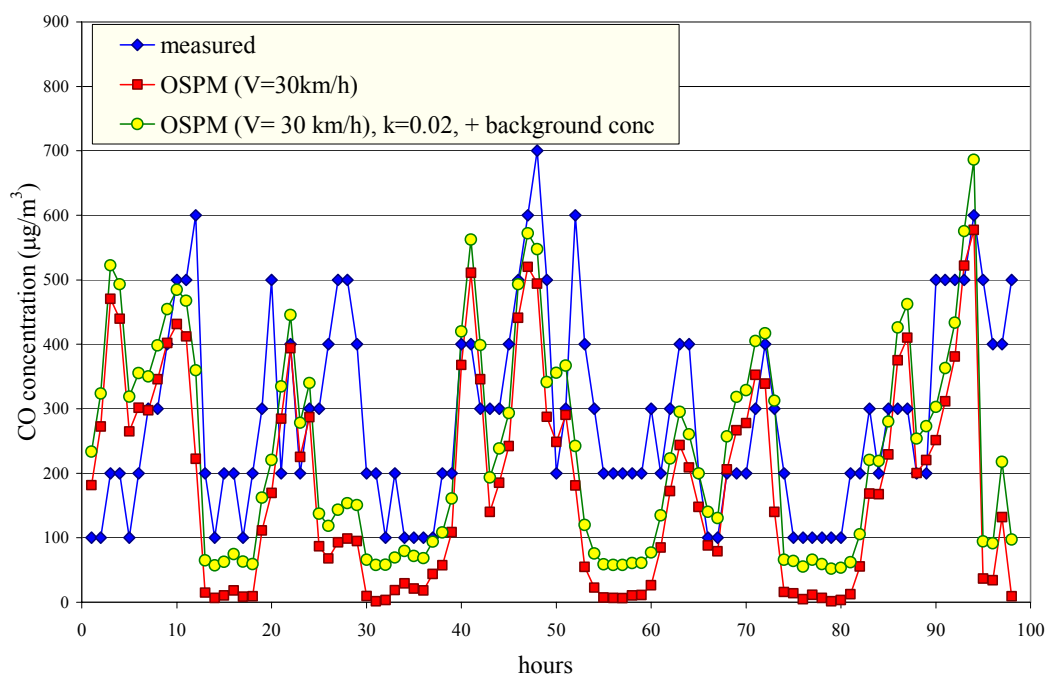

Figure 4: $\quad$ Measured and calculated CO concentrations.

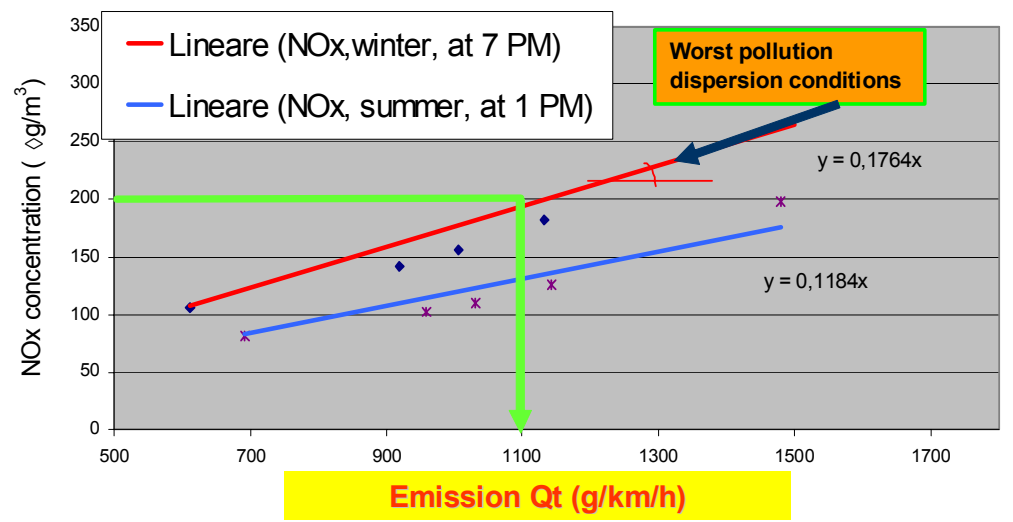

Figure 5: $\quad$ Maximum emissions in order to comply with limits.

This model was then used as a forecasting model to first of all evaluate how a limitation of the circulating vehicles (type of vehicles admitted) would influence the daily distribution of polluting concentrations. In more general terms, the maximum flows that the vehicle traffic altogether, however constituted, could emit in order to respect determined environmental quality levels in the worst atmospheric conditions in both the summer and winter periods were calculated 
and these results are reported in Figure 5. This latter appears, from the point of view of limiting strategy, to be the most significant response that the Public Organisation can consider as an aid for their intervention policies.

\section{Conclusions}

The three previously mentioned examples illustrate the applicability of the proposed methodology for different contexts and technologies. It is important to underline how, while this approach can be considered of a general nature, the results obtained for a certain situation cannot be extrapolated for other applications. While the correlations between technology and emissive flows can be considered general, the environmental effect of the latter is extremely sitespecific as it depends on local aspects such as meteorology, the street geometry or orography aspects and on synergy phenomena between different pollutants. Even the damage aspects depend on the particular situations, as the distribution of the population, the closeness of sensitive targets, the value that can be attributed to damage factors are all parameters that can certainly vary from situation to situation. Although it is important to state that in order to obtain a precise numerical result, or even only a parametric comparison of different situations, it is necessary to carry out a specific calculation procedure for each particular case according to the indicated procedure, the study of the operative guidelines however constitutes a procedure that can be generalised and which can draw some positive aspects from a specific study that has already been performed elsewhere. The thus described instrument can be considered to be an important step in territorial planning of some technological interventions; though not able to eliminate all the problems, it can offer the decision makers a reliable evaluation instrument.

\section{References}

[1] U.S. EPA, Office of Air Quality Planning and Standards, Revised Draft user's guide for the AMS/EPA regulatory model AERMOD, U.S. EPA, 2002.

[2] Brizio, E., Genon, G., Poggio, M., Results of atmospheric dispersion model for the localization of a MSW incinerator, W.I.T., ENVIROSOFT 2004.

[3] European Commission - DG XII, Externalities of Energy, volume 7: Methodology 1998 update, (EUR 19083 EN), Bruxelles, 1999.

[4] Brizio, E., Genon, G., Poggio, M., Comparison of locations for a MSW incineration plant, W.I.T., WASTE MANAGEMENT 2004.

[5] Politecnico di Torino, Analisi energetica e ambientale delprogetto di teleriscaldamento di Mondovi, 2005.

[6] Genon, G., Brizio, E., Modelling the effects of traffic emissions on the air quality, W.I.T., AIR POLLUTION 2005.

[7] Berkowicz, R., Olesen, H., Jensen, S., Operational Street Pollution Model, User's Guide to Win OSPM, National Environmental Research Institute, Denmark, 2003. 